517.53

doi:10.13108/2018-10-1-64

UDC 517.53

\title{
ON MEASURES GENERATING ORTHOGONAL POLYNOMIALS WITH SIMILAR ASYMPTOTIC BEHAVIOR OF THE RATIO AT INFINITY
}

\section{A.A. KONONOVA}

\begin{abstract}
We consider the influence of the measure perturbations on the asymptotic behavior of the ratio of orthogonal polynomials. We suppose that the absolutely continuous part of the measure is supported on finitely many Jordan curves. The weight function satisfies the modified Szegö condition.

The singular part of the measure consists of finitely many point masses outside the polynomial convex hull of the support of the absolutely continuous part of the measure. We study the stability of asymptotics of the ratio in the following sense:

$$
\frac{P_{\nu, n}(z)}{P_{\nu, n+1}(z)}-\frac{P_{\mu, n}(z)}{P_{\mu, n+1}(z)} \rightarrow 0, \quad n \rightarrow \infty .
$$

The problem is a generalization of the problem on compactness of the perturbation of Jacobi operator generated by the perturbation of its spectral measure. We find a condition necessary (or necessary and sufficient under some additional restriction) for the stability of the asymptotical behavior of the corresponding orthogonal polynomials. One of the main tools in the study are the Riemann theta functions.
\end{abstract}

Keywords: orthogonal polynomials, multivalued functions.

Mathematics Subject Classification: 30E15, 42C05

\section{INTRODUCTION}

The present work continues studying the stability of the asymptotics of the ratio of orthogonal polynomials under a perturbation of the orthogonality measure; the beginning of this study was published in paper [3].

For a finite Borel measure $\mu$ with a compact infinite support $E \subset \mathbb{C}$ we consider a sequence of polynomials with the leading coefficient equalling to one, $P_{\mu, n}(z)=z^{n}+\ldots$; these polynomials are orthogonal w.r.t. the measure $\mu$ :

$$
\int_{E} P_{\mu, n} \overline{P_{\mu, k}} d \mu=\alpha_{n} \delta_{n, k}, \quad \alpha_{n}>0 .
$$

We consider the measures concentrated on a finite set of Jordan curves $E=\cup_{k=0}^{p} E_{k} \subset \mathbb{C}$ and on a finite set of discrete masses located outside a polynomially convex hull of the set $E$.

It is well known that as $\operatorname{supp}(\mu) \subset \mathbb{R}$, the orthogonal polynomials satisfy the three-term recurrent relation:

$$
P_{n+1}(z)=\left(z-b_{n+1}\right) P_{n}(z)-a_{n}^{2} P_{n-1}(z) .
$$

A.A. Kononova, On measures generating ORThOgOnAl POLYNOMials With SIMILAR ASYMPTOTIC BEHAVIOR OF THE RATIO AT INFINITY.

(C) Kononova A.A. 2018.

The work is supported by RFBR (grant no. 17-51-150005).

Submitted March 9, $201 \%$. 
A three-diagonal matrix, whose entries are the coefficients of the recurrent relation

$$
J_{\mu}=\left(\begin{array}{ccccc}
b_{1} & a_{1} & 0 & 0 & \ldots \\
a_{1} & b_{2} & a_{2} & 0 & \ldots \\
0 & a_{2} & b_{3} & a_{3} & \ldots \\
\vdots & \vdots & \vdots & \vdots & \ddots
\end{array}\right),
$$

is called Jacobi matrix. If the set $\operatorname{supp}(\mu)$ is compact and infinite, the Jacobi matrix generates a bounded self-adjoint operator in $l^{2}(\mathbb{N})$, whose spectral measure is the measure $\mu$. It is known [2] that the operator $J_{\nu}$ is a compact perturbation of the operator $J_{\mu}$ (that is, the operator $J_{\nu}-J_{\mu}$ is compact) if and only if

$$
\frac{P_{\nu, n}(z)}{P_{\nu, n+1}(z)}-\frac{P_{\mu, n}(z)}{P_{\mu, n+1}(z)} \rightarrow 0, \quad n \rightarrow \infty
$$

the convergence is uniform on compact neighbourhood of the infinity.

In the general case $\operatorname{supp}(\mu) \subset \mathbb{C}$, as a generalization of the Jacobi matrix, we can consider Hessenberg matrices, that is, the matrices corresponding to the operator of multiplication by the independent variable in the space $L^{2}(\mu)$ in the basis of the corresponding orthogonal polynomials. It was shown in work [5] by B. Simanek that as $\operatorname{supp}(\mu) \subset \mathbb{C}$, condition (1) is equivalent to coinciding of the right limits of the corresponding Hessenberg matrices.

In work [3] there was found a condition for the measure $\nu$ sufficient (and necessary in some cases) for (1). In the present work, instead of condition (1) we consider a similar condition with a shift: for some fixed $l \in \mathbb{N}$

$$
\lim _{n \rightarrow \infty}\left(\frac{P_{n, \mu}(z)}{P_{n+1, \mu}(z)}-\frac{P_{n+l, \nu}(z)}{P_{n+l+1, \nu}(z)}\right)=0,
$$

where the convergence is uniform on compact neighbourhood of the infinity, and we also consider the case, when this condition holds for $n$ tending to infinity over a subsequence.

\section{MAIN DEFINITIONS AND AUXILIARY DATA}

In this section we provide main definitions and facts needed for the future exposition; for more details see [7]-[10].

2.1. Main notations. We say that a rectifiable curve belongs to the class $C^{2+}$ if the second derivatives of its coordinate functions, as functions of the arc length, satisfy the Lipschitz condition with some positive exponent.

Let $\Omega$ be a domain in the extended complex plane containing the infinity; the boundary of this domain consists of finitely many disjoint Jordan curves $E_{k}, k=0, \ldots, p$, belonging to the class $C^{2+}$ :

$$
\infty \in \Omega \subset \overline{\mathbb{C}}, \quad \partial \Omega=E:=\bigcup_{k=0}^{p} E_{k} .
$$

We consider the real Green function $g\left(z, z_{0}\right)$ with the logarithmic singularity at the point $z_{0} \in \bar{\Omega}$ (in the case $z_{0}=\infty$, instead of $g(z, \infty)$, we write $g(z)$ ), it can be defined by the following properties:

- $g\left(z, z_{0}\right)$ is harmonic in $\Omega \backslash z_{0}$;

- $g\left(z, z_{0}\right)-\log \left|z-z_{0}\right|^{-1}$ is harmonic in a neighbourhood of $z_{0}$, and as $z_{0}=\infty$, the function $g(z)-\ln |z|$ is harmonic in a neighbourhood of $\infty$;

- $\lim g\left(z, z_{0}\right)=0$ for a.e. $\zeta \in E$.

$$
\begin{aligned}
& z \rightarrow \zeta \\
& z \in \Omega
\end{aligned}
$$


Let $\left.\Phi\left(z, z_{0}\right)=\exp \left[g\left(z, z_{0}\right)+i \tilde{g}\left(z, z_{0}\right)\right]\right]$. We recall that the solution to the Dirichlet problem with a boundary function $f$ can be obtained by means of the normal derivative of the Green function:2

$$
h(z)=\frac{1}{2 \pi} \oint_{E} f(\zeta) \frac{\partial g(\zeta, z)}{\partial n_{\zeta}}|d \zeta|
$$

A logarithmic capacity of the set $E$ is the number $C(E)$ :

$$
\ln (C(E)):=-\lim _{z \rightarrow \infty}(g(z)-\log |z|) .
$$

A harmonic measure $\omega_{k}(z)(k=0, \ldots, p)$ is the solution to the Dirichlet problem in $\bar{\Omega}$ with the boundary function

$$
\chi_{k}(\zeta)= \begin{cases}1, & \zeta \in E_{k} \\ 0, & \zeta \in E \backslash E_{k}\end{cases}
$$

this solution reads as

$$
\omega_{k}(z):=\frac{1}{2 \pi} \oint_{E_{k}} \frac{\partial g(\zeta, z)}{\partial n_{\zeta}}|d \zeta|
$$

We let

$$
\Omega_{k}(z):=\frac{1}{2}\left(\omega_{k}(z)+i \tilde{\omega}_{k}(z)\right)
$$

2.2. Class of measures. In what follows we assume that

$$
\omega_{k}(\infty) \in \mathbb{Q}, \quad k=1, \ldots, p .
$$

We say that a weighted function $\rho(\zeta) \geqslant 0$ defined on $E$ and being such that

$$
\int_{E} \rho(\zeta)|d \zeta|<+\infty
$$

satisfies a modified Szegö condition if

$$
\oint_{E} \log \rho(\zeta) \frac{\partial g(\zeta)}{\partial n_{\zeta}}|d \zeta|>-\infty
$$

We define a class of measures $\mathcal{S}(E)$ as follows:

$$
\mu \in \mathcal{S}(E) \Leftrightarrow d \mu(\zeta)=\rho(\zeta)|d \zeta|+\sum_{k=1}^{N} A_{k} \delta_{z_{k}^{*}}(\zeta)
$$

where

1) $\rho(\zeta)$ satisfies the modified Szegö condition on $E$;

2) $z_{k}^{*} \in \Omega, A_{k}>0, k=1, \ldots, N$ and $\delta_{z}$ is the Dirac measure supported at the point $z$.

Given a measure $\mu \in \mathcal{S}(E)$ with a weight function $\rho(z)$, we define a locally analytic function $R_{\mu}(z)$ :

$$
R_{\mu}(z):=\exp (h(z)+i \tilde{h}(z)), \quad \text { where } \quad h(z)=\frac{1}{2 \pi} \oint_{E} \ln \rho(\zeta) \frac{\partial g(\zeta, z)}{\partial n_{\zeta}}|d \zeta|
$$

\footnotetext{
${ }^{1}$ Hereinafter $\tilde{h}(z)$ stands for the function harmonically conjugate with a harmonic function $h(z)$.

${ }^{2}$ Employing the symbol $\oint$, we mean that while integrating over a non-closed curve, it should be passed twice, once each side: $\oint_{E_{k}} F(\zeta)|d \zeta|=\int_{E_{k}^{+}} F_{+}(\zeta)|d \zeta|+\int_{E_{k}^{-}} F_{-}(\zeta)|d \zeta|$
} 
2.3. Multi-valuedness class. Given a multi-valued function $f$ defined in the domain $\Omega$ with a single-valued absolute value, we introduce the vector $\Gamma(f):=\left(\gamma_{1}(f), \ldots, \gamma_{p}(f)\right) \in \mathbb{T}^{p}$,

$$
\gamma_{k}(f):=\frac{1}{2 \pi} \Delta_{k} \arg f(\bmod 1)
$$

where $\Delta_{k} f$ is the increment of the function $f$ while passing around $E_{k}$. The vector $\Gamma(f)$ is called a multi-valuedness class of the function $f$.

We can show that $\Gamma\left(\Phi\left(z, z_{0}\right)\right)=\left(\omega_{1}\left(z_{0}\right), \ldots, \omega_{p}\left(z_{0}\right)\right)$. We introduce the notation:

$$
\Gamma_{n}:=\Gamma\left(\Phi^{-n}\right), \quad n \in \mathbb{Z} .
$$

2.4. Space $H^{2}(\Omega, \mu, \Gamma)$. We consider the $p$-dimensional real torus $\mathbb{T}^{p}$. Given a vector $\Gamma=$ $\left(\gamma_{1}, \ldots, \gamma_{p}\right) \in \mathbb{T}^{p}$ and a measure $\mu \in \mathcal{S}(E)$, we define a multi-valued Hardy space $H^{2}(\Omega, \mu, \Gamma)$ as follows:

$f \in H^{2}(\Omega, \mu, \Gamma)$ if and only if

- $f$ is locally analytic in $\Omega$ and has there a single-valued absolute value;

- the function $\left|f^{2} R_{\mu}\right|$ is subharmonic;

- $f\left(z_{k}^{*}\right)=0$

- $\frac{1}{2 \pi} \Delta_{k} \arg f \equiv \gamma_{k}(\bmod 1)$,

where $\Delta_{k} f$, as above, stands for the increment of the function $f$ while passing around $E_{k}$. The space $H^{2}(\Omega, \mu, \Gamma)$ is a Hilbert one with the scalar product

$$
(f, g)_{\mu}=\oint_{E} f(\zeta) \overline{g(\zeta)} d \mu(\zeta)
$$

We introduce a vector characteristic $\mathcal{J}(\mu) \in \mathbb{T}^{p}$ for a measure $\mu \in \mathcal{S}(E)$ with a weight $\rho(z)$ and masses $z_{j}^{*} \in \Omega, j=1, \ldots, N$, as follows:

$$
\mathcal{J}_{k}(\mu)=\frac{1}{4 \pi} \Delta_{k} \arg R_{\mu}(z)+\sum_{j=1}^{N} \omega_{k}\left(z_{j}^{*}\right)(\bmod 1), \quad k=1, \ldots, p .
$$

2.5. Riemann theta function. (see also [9].) We form a compact Riemann surface $\Omega_{\text {double }}$ of genus $p$ by the topological gluing of two copies $\Omega \cup E\left(\Omega_{+}, \Omega_{-}\right)$and identifying the points of $E$; the complex structure is continued to the "second" sheet $\Omega_{-}$via changing local parameters by complex conjugate ones. The functions analytic on $\Omega$ are continued on $\Omega_{\text {double }}$ :

$$
f(z):=\overline{f(\bar{z})}, \quad z \in \Omega_{-} .
$$

We define a homological basis on $\Omega_{\text {double }}$ as follows:

- $b$-cycles $b_{j}:=E_{j}, \quad j=1, \ldots, p$

- $a$-cycles are the curves $a_{j}, j=1, \ldots, p$ such that $a_{j} \cap \Omega_{+}$connects a fixed point $P \in E_{0}$ with $E_{j}$ and $a_{j} \cap \Omega_{-}$goes symmetrically over the second sheet $\Omega_{\text {double }}$ and $\cap_{j=1}^{p} a_{j}=\{P\}$. The surface obtained from $\Omega_{\text {double }}$ by a dissection along the homological basis is denoted by $\widetilde{\Omega}_{\text {double }}$. The differentials $d \Omega_{k}$ (Abelian differentials of the first kind) form a normed basis of the Abelian differentials of the surface $\Omega_{\text {double }}$. Let us calculate their periods along our homological basis:

$$
\oint_{\mathbf{a}_{j}} d \Omega_{k}(\zeta)=i B_{k, j}, \quad B_{k, j} \in \mathbb{R}
$$

where $B_{k, j}$ are the entries of some real positive definite matrix $B$. 
We define a theta function of several variables:

$$
\theta\left(u_{1}, u_{2}, \ldots, u_{p}\right)=\sum_{n_{1}, n_{2}, \ldots, n_{p} \in Z} \exp \left(-\pi \sum_{\mu=1}^{p} \sum_{\nu=1}^{p} B_{\mu, \nu} n_{\mu} n_{\nu}+2 \pi i \sum_{\nu=1}^{p} n_{\nu} u_{\nu}\right) ;
$$

the positive definiteness of the matrix $B$ with the entries $B_{k, j}$ ensures the convergence of the series. The theta function possesses the following properties:

$$
\begin{aligned}
& \theta\left(u_{1}, \ldots, u_{\mu}+1, \ldots, u_{p}\right)=\theta\left(u_{1}, \ldots, u_{\mu}, \ldots, u_{p}\right) \\
& \theta\left(u_{1}+i B_{1, \nu}, \ldots, u_{\mu}+i B_{\mu, \nu}, \ldots, u_{h}+i B_{p, \nu}\right)=e^{\pi B_{\nu, \nu}-2 \pi i u_{\nu}} \theta\left(u_{1}, \ldots, u_{\mu}, \ldots, u_{p}\right) .
\end{aligned}
$$

For an arbitrary vector $\left(\beta_{1}, \beta_{2}, \ldots, \beta_{p}\right)$, a Riemann theta function for the Riemann surface

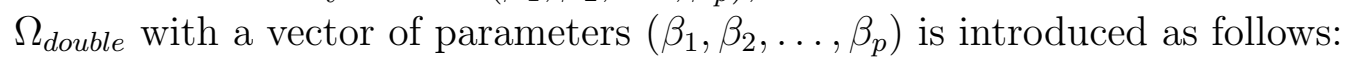

$$
\Theta(z)=\theta\left(\int_{P}^{z} d \Omega_{1}(\zeta)-\beta_{1}, \ldots, \int_{P}^{z} d \Omega_{p}(\zeta)-\beta_{p}\right)
$$

in each integral the integration is made over the same path. The Riemann theta function has exactly $p$ zeroes on $\widetilde{\Omega}_{\text {double }}$ or it vanishes identically. Its boundary values satisfy the following relations:

$$
\begin{array}{lll}
\Theta_{+}(\zeta)=\Theta_{-}(\zeta), & \zeta \in \mathbf{a}_{j}, & j=1, \ldots, p \\
\Theta_{+}(\zeta)=e^{\pi B_{j, j}-2 \pi i\left(\Omega_{j+}(\zeta)-\beta_{j}\right)} \Theta_{-}(\zeta), & \zeta \in \mathbf{b}_{j}, & j=1, \ldots, p .
\end{array}
$$

Let $z_{k}, k=1,2, \ldots, p$ be the zeroes of the Riemann theta function. The numbers

$$
k_{\nu} \equiv-\sum_{k=1}^{p} \int_{z_{0}}^{z_{k}} d \Omega_{\nu}(\zeta)+b_{\nu}
$$

are called Riemann constants and they are independent of the choice of $b_{\nu}$.

2.6. Extremal problem in space $H^{2}(\Omega, \mu, \Gamma)$. The linear functional mapping a function $F \in H^{2}(\Omega, \mu, \Gamma)$ into its value at a point $z_{0} \in \Omega$ (in particular, at infinity) is continuous. Therefore, in this space, there exists a reproducing kernel $K_{\mu, \Gamma}\left(z, z_{0}\right)$ :

$$
F\left(z_{0}\right)=\oint_{E} F(\zeta) \overline{K_{\mu, \Gamma}\left(\zeta, z_{0}\right)} d \mu(\zeta)
$$

Given a measure $\mu \in S(E)$ and a multi-valuedness class $\Gamma \in \mathbb{T}^{p}$, we define the function

$$
\psi_{\mu, \Gamma}(z)=\frac{K_{\mu, \Gamma}(z, \infty)}{K_{\mu, \Gamma}(\infty, \infty)} .
$$

It is easy to show that the function $\psi_{\mu, \Gamma}(z)$ possesses the following extremal property:

$$
\left\|\psi_{\mu, \Gamma}\right\|_{\mu}^{2}=\inf \left\{\|F\|_{\mu}^{2}, F \in H^{2}(\Omega, \mu, \Gamma),|F(\infty)|=1, F\left(z_{k}^{*}\right)=0, k=1, \ldots N\right\} .
$$

Indeed,

$$
1=|F(\infty)|^{2}=\left|\left(F(\cdot), K_{\mu, \Gamma}(\cdot, \infty)\right)_{\mu}\right|^{2} \leqslant\|F\|_{\mu}^{2} \cdot\left\|K_{\mu, \Gamma}(\cdot, \infty)\right\|_{\mu}^{2}=\|F\|_{\mu}^{2} \cdot K_{\mu, \Gamma}(\infty, \infty),
$$

and therefore,

$$
\left\|\psi_{\mu, \Gamma}\right\|_{\mu}^{2}=\frac{\left\|K_{\mu, \Gamma}(\cdot, \infty)\right\|_{\mu}^{2}}{\left|K_{\mu, \Gamma}(\infty, \infty)\right|_{\mu}^{2}}=\frac{1}{K_{\mu, \Gamma}(\infty, \infty)} \leqslant\|F\|_{\mu}^{2}
$$

One can show (see [7], [10]) that the zeroes of this function different from $z_{1}^{*}, \ldots, z_{N}^{*}$ belong to the convex hull of the set $E$. This function can be expressed in terms of the Riemann theta function [8], [10]:

$$
\psi_{\mu, \Gamma}(z)=\frac{K_{\mu, \Gamma}(z, \infty)}{K_{\mu, \Gamma}(\infty, \infty)}=\chi(z) \sqrt{\frac{R_{\mu}(\infty)}{R_{\mu}(z)}} \frac{\Theta(\Gamma, \mu, z)}{\Theta(\Gamma, \mu, \infty)} \prod_{k=1}^{N} \frac{\Phi\left(\infty, z_{k}^{*}\right)}{\Phi\left(z, z_{k}^{*}\right)}, n \in \mathbb{Z}_{+},
$$


where $\chi(z)$ is some single-valued function having no zeroes, depending only on the domain $\Omega$ and being independent both of $\Gamma$ and $\mu$; in paper [8], a formula for $\chi(z)$ was given but we do not need it in the present work. The symbol $\Theta(\Gamma, \mu, z)$ stands for the Riemann theta function with the vector of the parameters

$$
\beta_{j}(\Gamma, \mu)=d_{j}+\gamma_{j}+\mathcal{J}_{j}(\mu), \quad j=1,2, \ldots, p,
$$

where $\mathcal{J}_{j}(\mu)$ was defined above in $(3)$, and the constants $d_{j}$ are depend only on $\Omega$ and are independent both of $\Gamma$ and $\mu$; for more details see [8], [10].

We introduce the following notations:

$$
\Theta_{n, \mu}(z):=\Theta\left(\Gamma_{n}, \mu, z\right), \quad \psi_{n, \mu}(z):=\psi_{\mu, \Gamma_{n}} .
$$

2.7. Strong asymptotics of orthogonal polynomials. We shall need the following result, see [7], [10].

Theorem 1. Let $\mu \in \mathcal{S}(E)$ with a weight $\rho$ and discrete masses at the points $z_{k}^{*}, k=$ $1, \ldots, N$. Assume that polynomials $P_{n, \mu}$ with the unit leading coefficient are orthogonal w.r.t. the measure $\mu$. Then

$$
\begin{aligned}
& \text { 1) }\left\|P_{n, \mu}\right\|_{\mu}^{2} / C(E)^{2 n} \sim\left\|\psi_{n, \mu}\right\|_{\mu}^{2}, \quad n \rightarrow \infty ; \\
& \text { 2) } P_{n, \mu}(z)=C(E)^{n} \Phi^{n}(z)\left[\psi_{n, \mu}(z)+\epsilon_{n}(z)\right], \text { where } \epsilon_{n} \rightarrow 0 \quad \text { as } n \rightarrow \infty,
\end{aligned}
$$

uniformly on compact subsets $\Omega \backslash\left\{z_{1}^{*}, z_{2}^{*}, \ldots, z_{N}^{*}\right\}$.

\section{Formulation of PROBlem}

To each measure $\mu \in \mathcal{S}(E)$, a system of polynomials $P_{n, \mu}(z)=z^{n}+\ldots$ of a degree $n$ orthogonal w.r.t. $\mu$ corresponds to. We say that the polynomials $P_{n, \mu}$ and $P_{n, \nu}$ have a similar asymptotic behavior at infinity 1 if

$$
\frac{P_{n, \mu}(z)}{P_{n+1, \mu}(z)}-\frac{P_{n, \nu}(z)}{P_{n+1, \nu}(z)} \rightarrow 0, \quad n \rightarrow \infty
$$

uniformly on compact neighbourhoods of the infinity.

It was shown in [3] that the condition

$$
\mathcal{J}_{j}(\mu) \equiv \mathcal{J}_{j}(\nu)(\bmod 1), \quad j=1,2, \ldots, p,
$$

is sufficient for ensuring condition $(9)$ and it was proved that this condition is necessary in the case $p<4$. It was also shown that conditions $(10)$ and $(9)$ are equivalent for the measures $\mu \in \mathcal{S}(E)$ such that $E \subset \mathbb{R}$ (at that, the discrete component does not necessary belong to $\mathbb{R})$. It follows from the results of work [4] that if $E \subset \mathbb{R}$ possesses a discrete part consisting of countably many masses on $\mathbb{R}$ (the accumulation point must belong $E$ ) satisfying the modified Blaschke condition, under an obvious modification of $\mathcal{J}(\mu)$ for infinitely many masses, condition (10) is necessary and sufficient for condition (9) in the case of infinitely many discrete masses (see [4]).

In the present work, following B. Simanek (see [5]), we consider a more general condition instead of condition (9): for some fixed $l \in \mathbb{N}$,

$$
\lim _{n \rightarrow \infty}\left(\frac{P_{n, \mu}(z)}{P_{n+1, \mu}(z)}-\frac{P_{n+l, \nu}(z)}{P_{n+l+1, \nu}(z)}\right)=0,
$$

\footnotetext{
${ }^{1}$ It is important to note that the phrase "orthogonal polynomials have a similar asymptotic behavior at infinity" should be treated as a whole. This does not mean that for each measure the ratio possesses an asymptotics and these asymptotics coincide. There can be no asymptotics of the ratio for each measure.
} 
uniformly in compact neighbourhoods of the infinity. In this case we say that the orthogonal polynomials have same asymptotic behavior of the ratio with a shift.

\section{MAIN RESULTS}

For some multi-valuedness class $\widetilde{\Gamma} \in \mathbb{T}^{p}$ we define $\widetilde{\Gamma}_{-n}:=\widetilde{\Gamma}-\Gamma_{n}$.

Let $K_{\mu, \widetilde{\Gamma}_{-n}}\left(z, z_{0}\right)$ be a reproducing kernel in the space $H^{2}\left(\Omega, \mu, \widetilde{\Gamma}_{-n}\right)$ :

$$
\oint_{E} f(z) \overline{K_{\mu, \widetilde{\Gamma}_{-n}}\left(z, z_{0}\right)} d \mu(z)=f\left(z_{0}\right), \quad \forall f \in H^{2}\left(\Omega, \mu, \widetilde{\Gamma}_{-n}\right) .
$$

We observe that the function $f \in H^{2}\left(\Omega, \mu, \widetilde{\Gamma}_{-n}\right)$ satisfies $f(z) \Phi^{-1}(z) \in H^{2}\left(\Omega, \mu, \widetilde{\Gamma}_{-(n-1)}\right)$. Thus, we obtain a chain of embedded spaces

$$
\begin{aligned}
& \ldots \subset \Phi^{-n}(z) H^{2}\left(\Omega, \mu, \widetilde{\Gamma}_{-n}\right) \subset \Phi^{-n+1}(z) H^{2}\left(\Omega, \mu, \widetilde{\Gamma}_{-(n-1)}\right) \subset \ldots \\
& \ldots \subset \Phi^{-1}(z) H^{2}\left(\Omega, \mu, \widetilde{\Gamma}_{-1}\right) \subset H^{2}(\Omega, \mu, \widetilde{\Gamma}) .
\end{aligned}
$$

By the assumption, $\omega_{j}(\infty) \in \mathbb{Q}$, and therefore, $\Gamma_{q+k}=\Gamma_{k}$ for all $k$, where $q$ is the common denominator of the numbers $\omega_{j}(\infty) \in \mathbb{Q}$. Thus, the reproducing kernels also satisfy the periodicity condition w.r.t. the subscript:

$$
K_{\mu, \widetilde{\Gamma}_{-n}}\left(z, z_{0}\right)=K_{\mu, \widetilde{\Gamma}_{-(n+q)}}\left(z, z_{0}\right)
$$

We introduce the notation

$$
K_{n, \mu}(z, \infty):=K_{\mu, \widetilde{\Gamma}_{-n}}(z, \infty), \quad \phi_{n, \mu}(z):=K_{n, \mu}(z, \infty) \Phi^{-n}(z) .
$$

The next lemma generalizes Lemma 7.6 in work [6].

Lemma 1. The system of functions $\left\{\phi_{n, \mu}(z)\right\}_{n=0}^{\infty}$ is an orthogonal basis in the space $H^{2}(\Omega, \mu, \tilde{\Gamma})$.

Proof. a) Let $n<m$, then $\Phi^{n-m}(\infty)=0$. The definition of the reproducing kernel implies

$$
\begin{aligned}
\oint_{E} \phi_{n, \mu}(\zeta) \overline{\phi_{m, \mu}(\zeta)} d \mu(\zeta) & =\oint_{E} K_{n, \mu}(\zeta, \infty) \Phi^{n}(\zeta) \overline{K_{m, \mu}(\zeta, \infty) \Phi^{m}(\zeta)} d \mu(\zeta) \\
& =\oint_{E} K_{n, \mu}(\zeta, \infty) \Phi^{n-m}(\zeta) \overline{K_{m, \mu}(\zeta, \infty)} d \mu(\zeta)=K_{n, \mu}(\infty, \infty) \Phi^{n-m}(\infty)=0 .
\end{aligned}
$$

As $n=m$,

$$
\begin{aligned}
\oint_{E}\left|\phi_{n}(\zeta)\right|^{2} d \mu(\zeta) & =\oint_{E}\left|K_{n, \mu}(\zeta, \infty) \Phi^{n}(\zeta)\right|^{2} d \mu(\zeta)=\oint_{E}\left|K_{n, \mu}(\zeta, \infty)\right|^{2} d \mu(\zeta) \\
& =\left\|K_{n}(\cdot, \infty)\right\|^{2} \in(0, \infty)
\end{aligned}
$$

b) Let us prove that the system $\left\{\phi_{n, \mu}(z)\right\}_{n=0}^{\infty}$ is complete. Assume that there exists a non-zero vector $\phi \in H^{2}(\Omega, \mu, \tilde{\Gamma})$ such that

$$
\oint_{E} \phi(\zeta) \overline{\phi_{n, \mu}(\zeta)} d \mu(\zeta)=0 \quad n=0,1, \ldots
$$

The orthogonality of the vectors $\phi$ and $\phi_{0, \mu}$ and definition of the reproducing kernel yield that

$$
0=\oint_{E} \phi(\zeta) \overline{\phi_{0, \mu}(\zeta)} d \mu(\zeta)=\oint_{E} \phi(\zeta) \overline{K_{0, \mu}(\zeta, \infty)} d \mu(\zeta)=\phi(\infty)
$$


and therefore, $\phi(z) \Phi(z) \in H^{2}\left(\Omega, \mu, \tilde{\Gamma}_{1}\right)$ and $\phi \in \Phi^{-1} H^{2}\left(\Omega, \mu, \tilde{\Gamma}_{1}\right)$. It follows from the orthogonality of the vectors $\phi$ and $\phi_{1, \mu}$ that

$$
\begin{aligned}
0 & =\oint_{E} \phi(\zeta) \overline{\phi_{1, \mu}(\zeta)} d \mu(\zeta)=\oint_{E} \phi(\zeta) \overline{\Phi^{-1}(\zeta) K_{1, \mu}(\zeta, \infty)} d \mu(\zeta) \\
& =\oint_{E} \phi(\zeta) \Phi(\zeta) \overline{K_{1, \mu}(\zeta, \infty)} d \mu(\zeta)=(\phi \Phi)(\infty)
\end{aligned}
$$

Thus, the function $\phi \Phi$ vanishes at the point $\infty$, and therefore, $\phi \Phi^{2}(z) \in H^{2}\left(\Omega, \mu, \tilde{\Gamma}_{1}\right)$ and $\phi(z) \in \Phi^{-2}(z) H^{2}\left(\Omega, \mu, \tilde{\Gamma}_{1}\right)$. Arguing by induction, we obtain that an analytic in $\Omega$ function $\phi$ has a zero of order $m$ at infinity for arbitrary $m$. Therefore, there exists no function $\phi$ orthogonal to all functions $\phi_{n}$.

The following result allows us to "get rid" of the discrete component of a measure by changing the weight function so that the corresponding polynomials have the same asymptotic behavior of the ratio.

Lemma 2. Given a measure $\mu \in \mathcal{S}(E)$, there exists an absolutely continuous measure $\nu^{0} \in$ $\mathcal{S}(E)$ such that

$$
\frac{P_{n, \mu}(z)}{P_{n+1, \mu}(z)}-\frac{P_{n+l, \nu^{0}}(z)}{P_{n+l+1, \nu^{0}}(z)} \rightarrow 0, \quad n \rightarrow \infty,
$$

uniformly on compact neighbourhoods of the infinity.

This lemma is a simple corollary of the following stronger statement generalizing Corollary 3.2 in [11].

Theorem 2. Given two measures $\mu, \nu \in \mathcal{S}(E)$ and an arbitrary number $l \in \mathbb{N}$, there exists an absolutely continuous measure $\nu^{0} \in \mathcal{S}(E)$ such that

$$
\frac{P_{n, \mu}(z)}{P_{n+1, \mu}(z)}-\frac{P_{n+l, \nu^{0}}(z)}{P_{n+l+1, \nu^{0}}(z)} \rightarrow 0, \quad n \rightarrow \infty,
$$

uniformly in compact neighbourhoods of the infinity. At that, $\nu^{0}$ can be chosen so that on each connected component of the set $E$, the measures $\nu$ and $\nu^{0}$ differ by a constant factor:

$$
\left.\nu^{0}\right|_{E_{k}}=\left.C_{k} \cdot \nu\right|_{E_{k}}, \quad k=0, \ldots, p .
$$

Proof. We consider the function

$$
V(z):=\exp \left(\sum_{k=0}^{p} 2 \tau_{k} \Omega_{k}(z)\right)
$$

where the numbers $\tau_{k}$ are determined uniquely by the following system of equations (see [7]):

$$
\frac{1}{4 \pi} \sum_{k=0}^{p} \tau_{k} \Delta_{j} \tilde{\omega}_{k}=\mathcal{J}_{j}(\mu)-\mathcal{J}_{j}(\nu)-l \cdot \omega_{j}(\infty) ; \quad \sum_{k=0}^{p} \tau_{k}=0, \quad j=0, \ldots, p .
$$

It is easy to see that the absolute value of this function is constant on each connected component of $E$. Let

Then

$$
C_{k}:=|V(z)|=e^{\tau_{k}},\left.\quad \nu^{0}\right|_{E_{k}}=\left.C_{k} \cdot \nu\right|_{E_{k}}, \quad k=0, \ldots, p .
$$

$$
R_{\nu^{0}}=V \cdot R_{\nu}, \quad \mathcal{J}_{j}\left(\nu^{0}\right)=\frac{1}{4 \pi} \Delta_{E_{k}} \arg V(z)+\mathcal{J}_{j}(\nu)=\frac{1}{4 \pi} \sum_{k=0}^{p} \tau_{k} \Delta_{E_{j}} \tilde{\omega}_{k}+\mathcal{J}_{j}(\nu)=\mathcal{J}_{j}(\mu)
$$

and therefore,

$$
\mathcal{J}_{j}(\mu)-\mathcal{J}_{j}\left(\nu^{0}\right) \equiv 0(\bmod 1), \quad j=1, \ldots, p
$$


As it was shown in [3], this condition is sufficient for the validity of (12).

The main difficulty arising in proving conditions necessary for the behavior of ratio of orthogonal polynomials to be same is the possible cancelation of the zeroes of the functions $\Theta_{n, \mu}$, $n \in \mathbb{N}$ (see [3], [11]). In the next lemma we show that if such cancellation arises, such zeroes should belong to $E$.

Lemma 3. Consider the space $H^{2}(\Omega, \mu, \Gamma)$. The functions $\Theta_{n, \mu}, n \in \mathbb{N}$, can have a common zero only in the set $E$, that is, the existence of a common zero $z_{0} \in \Omega_{\text {double }}: \Theta_{n, \mu}\left(z_{0}\right)=0$ for all $n \in \mathbb{N}$ implies that $z_{0} \in E$.

Proof. By the measure $\mu$, we construct the measure $\nu^{0}$ as in Lemma 2. Since by the construction

$$
\mathcal{J}_{j}(\mu)-\mathcal{J}_{j}\left(\nu^{0}\right)=0(\bmod 1), \quad j=1, \ldots, p,
$$

then

$$
\Theta_{n, \mu}=\Theta_{n, \nu^{0}} .
$$

We split the proof into two parts. First we prove that $z_{0} \notin \Omega_{+}$, and in the second part we show that $z_{0} \notin \Omega_{-}$.

1) Assume that $z_{0} \in \Omega_{+}$.

Since the measure $\nu^{0}$ is absolutely continuous, as formula (8) shows, the zeroes of the functions $K_{\nu^{0}, \Gamma_{n}}$ coincide with the zeroes $\Theta_{n, \mu}$. Then, by the assumption, $K_{\nu^{0}, \Gamma_{n}}\left(z_{0}\right)=0$ for all $n \in \mathbb{N}$ and definition (11) implies that each function in the system $\phi_{n}=\Phi^{-n} K_{\nu^{0}, \Gamma_{n}}$ vanishes at the point $z_{0} \in \Omega$. As it was shown in Lemma 1, the functions $\phi_{n}, n \geqslant n_{0}$, forms a basis in the space $H^{2}\left(\Omega, \nu^{0}, \Gamma_{n_{0}}\right)$ for each $n_{0} \in \mathbb{N}$. However, as it was shown in [7], one can construct a function $V_{n_{0}} \in H^{2}\left(\Omega, \nu^{0}, \Gamma_{n_{0}}\right)$ not vanishing in $\Omega$ :

$$
V_{n_{0}}(z):=\exp \left(\sum_{k=0}^{p} 2 \tau_{k} \Omega_{k}(z)\right)
$$

where the numbers $\tau_{k}$ are uniquely determined by the following system of equations (a more detailed construction of the functions $V_{\Gamma_{n_{0}}}$ can be found in [7]):

$$
\sum_{k=0}^{p} \tau_{k} \Delta_{E_{j}} \tilde{\omega}_{k}=\gamma_{j} ; \quad \sum_{k=0}^{p} \tau_{k}=0, \quad j=0, \ldots, p .
$$

The obtained contradiction shows that $z_{0} \notin \Omega_{+}$.

The proven statement is also implied by the Ambroladze theorem [1].

2) Now we consider the case $z_{0} \in \Omega_{-}$.

We recall that the extremal function $\psi_{n, \nu^{0}}$ has the same zeroes as the function $\Theta_{n, \nu^{0}}(8)$. In Theorem 6.2 in [7], there was provided a system of equation determining uniquely the zeroes of the function $\psi_{n, \nu^{0}}$ :

$$
\begin{aligned}
& \sum_{j=1}^{p}\left(\widetilde{\omega}_{k}\left(z_{j}^{0}\right)-\widetilde{\omega}_{k}\left(z_{j}^{*}\right)\right)=\sum_{l=1}^{p} m_{l} \Delta_{k} \widetilde{\omega}_{l} \\
& \frac{1}{2} \sum_{j=1}^{p} \epsilon_{j} \omega_{k}\left(z_{j}^{0}\right)=\mathcal{J}_{k}\left(\nu_{0}\right)-\frac{\omega_{k}(\infty)-1}{2}-n \omega_{k}(\infty)(\bmod 1)
\end{aligned}
$$

where $z_{0}^{*}$ are finite zeroes of $G^{\prime}(z) ; m_{l}$ are arbitrary natural numbers. The parameters $\epsilon_{j}= \pm 1$ and the points $z_{j}^{0}$ are determined uniquely by the system and at that, the numbers $\epsilon_{j}$ serve for indicating the sheet of the Riemann surface, on which one should put the corresponding point $z_{j}^{0}$ : if $\epsilon_{j}=1$, then $z_{j}^{0} \in \Omega_{+}$, and if $\epsilon_{j}=-1$, then $z_{j}^{0} \in \Omega_{-}$. 
By our assumption, one of the points $z_{j}^{0}$ is the point $z_{0}$, and the corresponding parameter is equal to -1 . Without loss of generality, we suppose that $z_{1}^{0}=z_{0}, \epsilon_{1}=-1$. To the right hand side of the second equation in system (13), we add the term $2 \omega_{k}\left(z_{1}^{0}\right)$. It is obvious that this equation is also satisfied by the same system of the points with the same parameters $\epsilon_{j}$, $j=2, \ldots, p$, and the parameter $\epsilon_{1}=1$.

On the other hand, the same system of equations can be obtained by solving the extremal problem for the weight function $\widetilde{\nu}_{0}$ such that

$$
\mathcal{J}_{k}\left(\nu_{0}\right)=\mathcal{J}_{k}(\nu)+2 \omega_{k}\left(z_{0}\right), \quad k=0, \ldots, p
$$

the construction of such function reproduces that in the proof of Theorem 2. Thus, we obtain that the extremal functions $\psi_{n, \nu_{0}}$, and therefore, $\Theta_{n, \nu_{0}}$, have the common zero at a point in the domain $\Omega_{+}$. But, as it follows from Statement (1), this is impossible.

Theorem 3. Let $\omega_{k}(\infty) \in \mathbb{Q}, k=1, \ldots, p, \mu, \nu \in \mathcal{S}(E)$ and

$$
\lim _{n \rightarrow \infty}\left(\frac{P_{n, \mu}(z)}{P_{n+1, \mu}(z)}-\frac{P_{n+l, \nu}(z)}{P_{n+l+1, \nu}(z)}\right)=0
$$

for some fixed $l \in \mathbb{N}$; the convergence is uniform on compact neighbourhoods of the infinity.

Then

$$
\mathcal{J}_{j}(\mu) \equiv \mathcal{J}_{j}(\nu)-l \cdot \omega_{j}(\infty)\left(\bmod \frac{1}{2}\right), \quad j=1,2, \ldots, p
$$

Proof. We assume that

$$
\frac{P_{n, \mu}(z)}{P_{n+1, \mu}(z)}-\frac{P_{n+l, \nu}(z)}{P_{n+l+1, \nu}(z)} \rightarrow 0, \quad n \rightarrow \infty
$$

uniformly in compact neighbourhoods of the infinity. Then

$$
\frac{\psi_{n, \mu}(z)}{\psi_{n+1, \mu}(z)}-\frac{\psi_{n+l, \nu}(z)}{\psi_{n+l+1, \nu}(z)} \rightarrow 0, \quad n \rightarrow \infty
$$

Employing representations (8), we obtain

$$
\frac{\Theta_{n, \mu}(z) \Theta_{n+1, \mu}(\infty)}{\Theta_{n+1, \mu}(z) \Theta_{n, \mu}(\infty)}-\frac{\Theta_{n+l, \nu}(z) \Theta_{n+l+1, \nu}(\infty)}{\Theta_{n+l+1, \nu}(z) \Theta_{n+l, \nu}(\infty)} \rightarrow 0, \quad n \rightarrow \infty
$$

Let $q \in \mathbb{N}$ be a common denominator of the numbers $\omega_{k}(\infty) \in \mathbb{Q}, k=1, \ldots, p$, then $\Gamma_{n}=\Gamma_{n+q}$ for all $n \in \mathbb{N}$ and therefore, $\Theta_{n, \mu}=\Theta_{n+q, \mu}$. Then the asymptotic formulae imply that

$$
\frac{\Theta_{r, \mu}(z) \Theta_{r+1, \mu}(\infty)}{\Theta_{r+1, \mu}(z) \Theta_{r}, \mu(\infty)}=\frac{\Theta_{r+l, \nu}(z) \Theta_{r+l+1, \nu}(\infty)}{\Theta_{r+l, \nu}(z) \Theta_{r+l+1, \nu}(\infty)} \quad r \in \mathbb{N}, \quad r \leqslant q-1 .
$$

If none of $p$ zeroes of the theta function $\Theta_{\mu, r}(z)$ cancels out with a zero of the function $\Theta_{\mu, r+1}(z)$, then the zeroes of $\Theta_{\mu, r}(z)$ and $\Theta_{\nu, r+l}(z)$ should coincide. If for some $z_{\mu} \in \widetilde{\Omega}$ we have $\Theta_{\mu, r}\left(z_{\mu}\right)=\Theta_{\mu, r+1}\left(z_{\mu}\right)$, then the cancelation occurs also in the left hand side of identity (16). Let $\Theta_{\nu, r+l}\left(z_{\nu}\right)=\Theta_{\nu, r+l+1}\left(z_{\nu}\right)$ and $z_{\mu} \neq z_{\nu}$. By Lemma 3, if $z_{\mu} \notin E$, there exists $r_{0}$ such that $\Theta_{\mu, r_{0}}\left(z_{\mu}\right) \neq 0$. But then the corresponding identity of the ratios of theta functions (16) implies that $\Theta_{\nu, r_{0}+l}\left(z_{\nu}\right) \neq 0$ and $z_{\mu}=z_{\nu}$. 
We write the expressions for the Riemann constants of the surface $\Omega_{\text {double }}$ in two ways (see (7)):

$$
\begin{aligned}
k_{j} & \equiv-\sum_{k=1}^{p} \int_{z_{0}}^{z_{k, \mu}} d \Omega_{j}(\zeta)+d_{j}-r_{0} \omega_{j}(\infty)+\mathcal{J}_{j}(\mu) \\
& \equiv \sum_{k=1}^{p} \int_{z_{0}}^{z_{k, \nu}} d \Omega_{j}(\zeta)+d_{j}-\left(r_{0}+l\right) \omega_{j}(\infty)+\mathcal{J}_{j}(\nu),
\end{aligned}
$$

where $z_{k, \mu}$ and $z_{k, \nu}$ are the zeroes of the functions $\Theta_{\mu, r}(z)$ and $\Theta_{\nu, r}(z)$, repsectively. And since all zeroes of the functions $\Theta_{\mu, r}(z)$ and $\Theta_{\nu, r}(z)$ coincide except, probably, those in the set $E$, we obtain (15).

Let us formulate a stronger statement being a simple corollary of the above arguing for the case, when the functions $\Theta_{n, \mu}, \Theta_{n+1, \mu}$ have no common zeroes in $E$ for some subsequence. Since there are no coinciding zeroes, in this case, the identity in condition (15) holds modulo 1.

Theorem 4. Let $\omega_{k}(\infty) \in \mathbb{Q}, k=1, \ldots, p, \mu, \nu \in \mathcal{S}(E)$. If for some infinite subset of natural numbers $\Lambda \subset \mathbb{N}$ the functions $\Theta_{n, \mu}$ and $\Theta_{n+1, \mu}$ have no common zeroes in $E$ for each $n \in \Lambda$, that is, $\Theta_{n, \mu}^{2}(z)+\Theta_{n+1, \mu}^{2}(z) \neq 0$ for all $z \in E$, for all $n \in \Lambda$, then the following conditions are equivalent:

- for some fixed $l \in \mathbb{N}$

$$
\frac{P_{n, \mu}(z)}{P_{n+1, \mu}(z)}-\frac{P_{n+l, \nu}(z)}{P_{n+l+1, \nu}(z)} \rightarrow 0, \quad n \rightarrow \infty, \quad n \in \Lambda,
$$

uniformly in compact neighbourhoods of the infinity;

- $\mathcal{J}_{j}(\mu) \equiv \mathcal{J}_{j}(\nu)-l \cdot \omega_{j}(\infty)(\bmod 1), j=1,2, \ldots, p$;

- for some fixed $l \in \mathbb{N}$

$$
\frac{P_{n, \mu}(z)}{P_{n+1, \mu}(z)}-\frac{P_{n+l, \nu}(z)}{P_{n+l+1, \nu}(z)} \rightarrow 0, \quad n \rightarrow \infty,
$$

uniformly in compact neighbourhoods of the infinity.

Thus, under the assumptions of Theorem 4, the preservation of the asymptotics of the ratio of two orthogonal polynomials with a shift is equivalent to preservation the asymptotic behavior over some subsequence.

The conditions of the measure in Theorem 4 are implicit. As an example of the measures obeying this statement, we provide the following corollary.

Corollary 1. If $\omega_{k}(\infty) \in \mathbb{Q}, k=1, \ldots, p, \mu, \nu \in \mathcal{S}(E)$, and the measure $\mu$ is invariant w.r.t. the rotations by the angle $\frac{2 \pi}{p+1}$, then the assumptions of Theorem 4 hold.

Proof. The uniqueness of the extremal function $\psi_{\mu, k}$ and the invariance of the measure w.r.t. the rotations imply that if some zero $z^{*}$ of the extremal function $\psi_{\mu, k}$ belongs to a connected component $E_{j}$, then the points obtained by rotation by the angle $\frac{2 \pi}{p+1}$ are also the zeroes of the extremal function. But this is impossible since the extremal function has at most $p$ zeroes, while the number of the connected components of the set $E$ is equal to $p+1$.

The author expresses his gratitude to the referee for valuable remarks. 


\section{BIBLIOGRAPHY}

1. A. Ambroladze. On exceptional sets of asymptotic relations for general orthogonal polynomials // J. Approx. Theory. 82:2, 257-273 (1995).

2. B. Beckermann. Complex Jacobi matrices // J. Comput. Appl. Math. 127:1-2, 17-65 (2001).

3. A.A. Kononova. Stability of ratio asymptotics of orthogonal polynomials under some class of measure perturbations // Acta Sci. Math. (Szeged). 81:1-2, 133-143 (2015).

4. F. Peherstorfer, P. Yuditskii. Asymptotic behavior of polynomials orthonormal on a homogeneous set // J. d'Anal. Math. 89:1, 113-154 (2003).

5. B. Simanek. Relative asymptotics for general orthogonal polynomials // Michigan Math. J. 66:1, 175-193 (2017).

6. M. Sodin, P. Yuditskii. Almost periodic Jacobi matrices with homogeneous spectrum, infinite dimensional Jacobi inversion, and Hardy spaces of character-automorphic functions // J. Geom. Anal. 7:3, 387-435 (1997).

7. H. Widom. Extremal polynomials associated with a system of curves in the complex plane // Adv. Math. 3:2, 127-232, (1969).

8. A.I. Aptekarev. Asymptotic properties of polynomials orthogonal on a system of contours, and periodic motions of Toda lattices // Matem. Sborn. 125(167):2(10), 231-258 (1984). [Math. USSR-Sb. 53:1, 233-260 (1986).]

9. B.A. Dubrovin. Theta functions and non-linear equations // Uspekhi Matem. Nauk. 36:2(218), 11-80 (1981). [Russ. Math. Surv. 36:2, 11-92 (1981).]

10. V.A. Kalyagin, A.A. Kononova. On the asymptotics of polynomials orthogonal with respect to a measure with atoms on a system of arcs // Alg. Anal. 21:2, 71-91 (2009).

11. A.A. Kononova. On compact perturbations of finite-zone Jacobi operators // Zap. Nauch. Sem. POMI. 366, 84-101 (2009). [J. Math. Sci. 165:4, 473-482 (2010).]

Anna Alexandrovna Kononova,

Saint Petersburg University,

Stary Peterhof, Universitetstky av. 28,

198504, Saint-Petersburg, Russia

E-mail: a.kononova@spbu.ru 